\title{
Coherent Radio Bursts from RS CVn Binaries
}

\author{
Elena Franciosini ${ }^{1}$, Stephen M. White ${ }^{2}$ \\ 1 Department of Astronomy and Space Science, University of Florence, \\ Largo E. Fermi 5, I-50125 Florence, Italy \\ 2 Department of Astronomy, University of Maryland, College Park, MD 20742, USA
}

We present VLA observations of RS CVn binaries which indicate that the low-frequency polarization inversion usually ascribed to the quiescent radio emission of these systems is in fact due to the presence, much of the time, of a coherent burst emission. Fig. 1 shows two examples of this coherent emission in the behaviour of the light curves of HR 1099 in left and right circular polarization at $1.4 \mathrm{GHz}$. The emission is highly circularly polarized, with one polarized component steady or slowly varying in a way similar to the higher frequency emission (Fig. 1a), while the other shows rapid fluctuations on timescales of $\sim 10 \mathrm{~s}$ and a slower modulation on longer timescales. The similarity of the time profiles in two $50 \mathrm{MHz}$-wide sidebands $30 \mathrm{MHz}$ apart indicates that the fluctuating component is broadband; moreover, the sense of polarization at $1.4 \mathrm{GHz}$ is opposite to that of the quiescent emission at higher frequencies (attributed to gyrosynchrotron emission), which is exactly the behaviour attributed to the $1.4 \mathrm{GHz}$ quiescent emission of these systems by Mutel et al. (1987).

The rapid fluctuations and high polarization are characteristic of a coherent emission process. We argue that a combination of two sources of emission is generally present at $1.4 \mathrm{GHz}$ : a very highly (possibly $100 \%$ ) polarized coherent component, and a weakly-polarized, steady gyrosynchrotron component. Since the polarization at higher frequencies should be the $\mathrm{x}$-mode of (optically thin) gyrosynchrotron emission, we infer that the low frequency polarization probably represents the o-mode. The observed characteristics of the emission then suggest that the most likely mechanism for the highly-polarized component is plasma emission at the fundamental of the plasma frequency. Such plasma emission is relatively rare on the Sun at $1.4 \mathrm{GHz}$, but could occur in the hotter coronae of RS CVn systems because the higher temperature reduces the free-free opacity which is thought to absorb $1.4 \mathrm{GHz}$ plasma emission on the Sun.

These observations suggest a different interpretation for the reversal in the sense of polarization at low frequencies previously attributed to the quiescent radio emission of RS CVn binary systems, which has proven very difficult to explain in terms of plausible gyrosynchrotron models (see White \& Franciosini, 1995). We propose that a coherent o-mode emission is frequently present also during low-activity periods, when it may be too weak to be distinguished from the gyrosynchrotron component. The gyrosynchrotron component is probably 

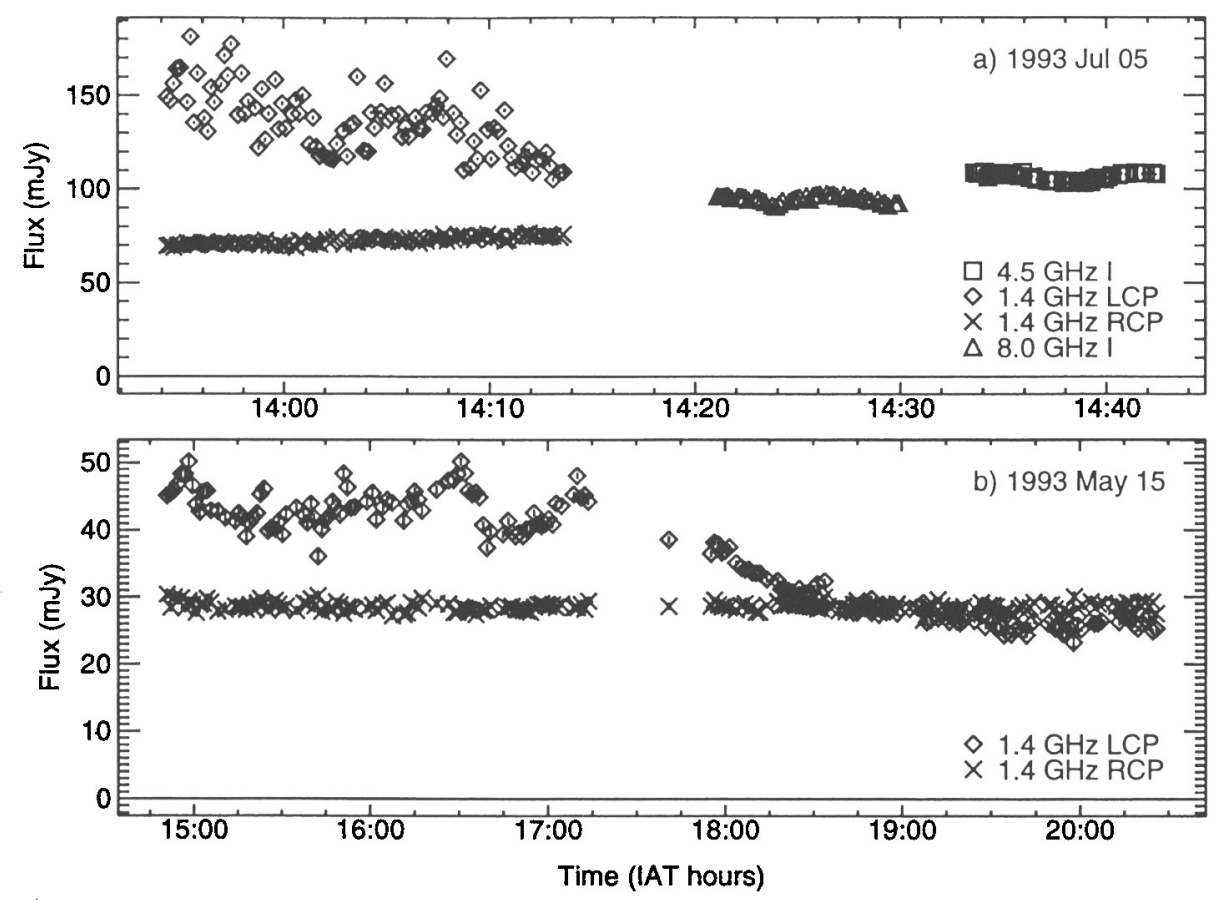

Fig. 1. The time profiles of right ( $R$ ) and left $(\mathrm{L})$ circular polarization at $1.4 \mathrm{GHz}$ during a VLA observation of HR 1099 on $1993 \mathrm{July} 5$ (upper) and 1993 May 15 (lower). In the first case the total intensity (I) in subsequent scans at $4.5 \mathrm{GHz}$ and $8.1 \mathrm{GHz}$ is also plotted. Error bars of $\pm \sigma$ are plotted on all points.

weakly polarized, so that the polarization of the integrated emission is dominated by the coherent component. We have some data suggesting that the true polarization of the quiescent emission at $1.4 \mathrm{GHz}$ is in the sense of the $\mathrm{x}$-mode as at higher frequencies. An example is shown in Fig. $1 \mathrm{~b}$, where the steady emission observed after the decay of the rapidly-varying coherent component is clearly polarized in the opposite sense.

The long duration of the observed coherent emission ( $20 \mathrm{~min}$ - several hours) together with the rapid variability $(\sim 10 \mathrm{~s})$ suggest that there must be a continuous supply of energetic electrons to the source, which requires a continuous acceleration mechanism and therefore also a continuous energy release in the corona of these stars. Such emission may therefore be evidence for steady, rather than sporadic, sites of energy release in the coronae of active stars.

\section{References}

Mutel R.L., Morris D.H., Doiron D.J., Lestrade J.-F., 1987, AJ 93, 1220

White S.M., Franciosini E., 1995, ApJ (in press) 